\title{
Design de interação integrado ao design instrucional: a modelagem de um ambiente de MOOC por meio da MoLIC
}

\author{
Filipe A. Garrido ${ }^{1}$, Beatriz B. do Rêgo' ${ }^{1}$, Ecivaldo de Souza Matos ${ }^{1}$ \\ ${ }^{1}$ Instituto de Computação (IC) - Universidade Federal da Bahia (UFBA) \\ Salvador - BA - Brasil \\ \{filipe.garrido, beatrizbr, ecivaldo\}@ufba.br
}

Resumo. A complexidade em harmonizar o discurso dos diversos agentes envolvidos na criação de um curso MOOC (acrônimo para Massive Open Online Courses) tem sido um desafio compartilhado entre o design de interação e o design instrucional, em que comunicação entre sujeitos é essencial para a qualidade do ensino e da aprendizagem. Um dos aspectos desse desafio está na necessidade de modelar a comunicação entre os interlocutores educacionais mediatizadas pelas interfaces de um sistema. Desse modo, considerando a Modeling Language for Interaction as Conversation (MoLIC) como uma ferramenta epistêmica de suporte à modelagem de interação (humano-computador), este artigo apresenta os resultados de uma investigação acerca da aplicação/adequação da MoLIC à modelagem de sistemas educacionais, especificamente uma plataforma e curso(s) MOOC, com foco na relação entre o design instrucional e o design de interação, resultando na adição de elementos específicos do domínio educacional e as intenções de design à MoLIC.

\section{Introdução}

A comunicação é fundamental para conexão entre os diferentes agentes que farão parte do cenário pedagógico [Freire 1983], podendo ser compreendida como um elemento de humanização do ser por permitir o diálogo desse indivíduo com o mundo e seus pares.

Na concepção de sistemas computacionais colaborativos para uso educacional, a comunicação entre os designers (instrucionais e de interação) é fundamental. Ao considerar a comunicabilidade como atributo de qualidade, a interação entre os interlocutores deve ser adequada para que os processos de ensino e de aprendizagem aconteçam, pois o objetivo desses sistemas é a construção de conhecimento [Melo, Saldanha e Wernz 2012].

Dentre os diferentes ambientes educacionais, os Massive Open Online Courses (MOOC), surgidos no contexto de educação a distância por meio da internet, são considerados um grande expoente para a democratização do conhecimento sem barreiras para milhares, talvez milhões, de pessoas ao redor do mundo [Aguaded 2013; Carey 2012; Martin 2012].

Zhao et al. (2020) indicam que um dos problemas dos MOOC é sua alta taxa de evasão. Os autores indicam que um dos possíveis fatores que influenciam essa evasão é a baixa interação entre os participantes que se sentem isolados nos ambientes propícios para o diálogo, como fóruns de discussão e chats [Do Rêgo, Garrido e Matos 2017; Do Rêgo et al. 2019]. Reforçando nosso argumento sobre a importância da comunicação 
entre sujeitos nos processos de ensino e de aprendizagem. Por isso, um dos desafios na modelagem de um ambiente de MOOC é a necessidade de promover comunicabilidade adequada aos agentes, sistema-estudante e estudante-estudante.

A modelagem da interação é fortemente relacionada ao domínio da temática, como o domínio educacional no caso dos MOOC [Carvalho et al. 2019]. Desse modo, analisou-se como integrar o design instrucional à modelagem de interação de sistemas específicos, como os MOOC. Para isso, adotou-se a Modeling Language for Interaction as Conversation (MoLIC) [Paula 2003; Silva 2005] como ferramenta epistêmica de suporte à modelagem da interação (humano-computador).

Neste artigo são apresentados os resultados de uma investigação acerca da aplicação/adequação da MoLIC na modelagem de sistemas educacionais, especificamente uma plataforma e curso(s) MOOC, com foco na relação entre o design instrucional e o design de interação.

O texto está organizado em seis seções. A Seção 2, nomeada de Fundamentação Teórica, apresenta aspectos conceituais da Engenharia Semiótica, MoLIC e Design Instrucional. Na Seção 3 está descrita a metodologia de pesquisa e na Seção 4 os resultados. Na Seção 5 há uma discussão acerca da inserção de novos elementos na MoLIC. Por fim, na Seção 6 estão as conclusões, seguidas dos agradecimentos e referências utilizadas neste estudo.

\section{Fundamentação Teórica}

\subsection{Engenharia Semiótica}

A Engenharia Semiótica (EngSem) é uma teoria explicativa de Interação Humano-Computador (IHC) [De Souza 2005]. Nessa perspectiva, é uma teoria que compreende fenômenos envolvidos no design, avaliação, uso e compreensão de um sistema interativo, focalizada nos fenômenos de significação e comunicação.

A partir da perspectiva da EngSem, designers e usuários passam a ter o mesmo papel, o de interlocutores em um processo comunicativo global [De Souza 2005]. Designers, em tempo de interação, comunicam suas intenções e concepções de design aos usuários por intermédio de signos dispostos na interface (layout gráfico, imagens, sons, textos).

Por meio da EngSem, pesquisadores instrumentalizam-se para entender, explicar e descrever fenômenos de interação baseados no diálogo entre designers e usuários, em que a comunicabilidade é conceito fundamental. A comunicabilidade pode ser definida como a propriedade de transmitir ao usuário, eficaz e eficientemente, as intenções e princípios de interação que guiaram o design do sistema [Prates, De Souza e Barbosa 2000].

A EngSem possui dois importantes métodos, o Método de Inspeção Semiótica (MIS) [De Souza 2005] e o Método de Avaliação de Comunicabilidade (MAC) [De Souza, Leitão 2009], ambos úteis para avaliação da qualidade da interação. Além disso, a EngSem inspirou a concepção da linguagem MoLIC para auxiliar no design de interação. 


\subsection{MoLIC}

A MoLIC (Modeling Language for Interaction as Conversation) foi proposta em 2003 como uma linguagem de modelagem para designers de IHC, com o objetivo de modelar a interação usuário-sistema, fundamentando-se na EngSem [Paula 2003].

Desde a sua proposta inicial, várias aplicações foram modeladas com MoLIC para diferentes domínios e contextos [Carvalho et al. 2019]. Algumas extensões foram propostas e desencadearam um esforço para sua revisão abrangente, o que resultou na $2^{\mathrm{a}}$ edição da MoLIC [Silva 2005; Silva e Barbosa 2007].

A MoLIC evidencia a interação sob o ponto de vista do designer, por intermédio do seu preposto (sistema), em diálogo com o usuário, ambos interlocutores em uma conversa. O design na perspectiva da MoLIC inicia-se geralmente com a definição dos perfis, papéis, objetivos do usuário. Essa definição é representada nos cenários de interação e os signos são explicitados no contexto dos cenários [Paula 2003; Silva 2005; Silva e Barbosa 2007; Barbosa e Silva 2010].

Na primeira etapa do projeto de interação, chamada de estruturação da conversa, o designer tem o objetivo de pormenorizar todas as possíveis conversas que ocorrerão entre os usuários e o preposto do designer em um diagrama de interação [Barbosa e Silva 2010]. O diagrama de interação representa como os objetivos do usuário serão alcançados durante a interação com o sistema em formato de um esquema visual conciso.

As representações dos elementos diagramáticos básicos para composição de um diagrama de interação da MoLIC são: (a) ponto de entrada, (b) ponto de saída, (c) ponto de contato com outro (papel de) usuário, (d) ponto de contato com sistema externo, (e) acesso ubíquo, (f) processamento do sistema, (g) fala de transição, (h) fala de transição para recuperação de ruptura, (i) fala de influência, (j) fala de transição com abertura de conversa sobre meta final, (l) fechamento de conversa sobre meta final, (m) bifurcação, (n) cena, (o) cena em sua forma mínima e (p) cena vazia (ver Figura 1) [Araujo 2008].

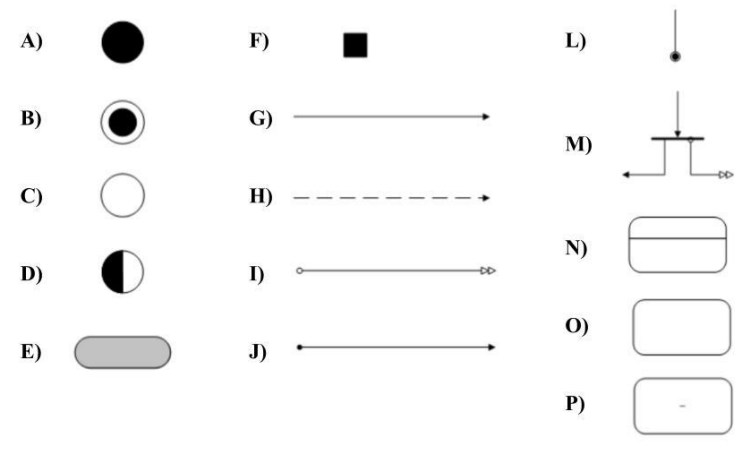

Figura 1: Elementos diagramáticos da MoLIC [Araujo 2008]

Segundo Silva (2005), a segunda etapa do projeto de interação, detalhamento da conversa, destaca que o designer nesse momento concentra seus esforços em detalhar a interação, ou seja, apresentar os diálogos e preocupar-se com as falas de transição para recuperação de breakdowns. $\mathrm{O}$ autor destaca ainda que existem quatro passos envolvendo a especificação dos diálogos: a estruturação dos diálogos na cena, as possíveis restrições sobre o rumo da conversa (falas de transição que pressupõem um 
conjunto de diálogos travados na cena de origem), definição e estruturação dos signos envolvidos nos diálogos e, por último, o detalhamento da ontologia de signos.

A MoLIC é uma linguagem para modelagem de interação que não possui relação com as especificações técnicas do sistema interativo modelado. Por esse motivo, em domínios específicos, como o educacional, pode existir a necessidade de interligação com outras ciências de design, como o design instrucional, para o alcance de um resultado mais adequado para o sistema em desenvolvimento.

\subsection{Design Instrucional e MOOC}

O design instrucional (DInst) é a atividade de criação do currículo pedagógico que se destina a produzir resultados de aprendizagem específicos, baseados não apenas na pesquisa pedagógica, mas também nas práticas pedagógicas contemporâneas [Filatro 2004].

Entretanto, não há relação direta do DInst com o desenvolvimento de software educacional ou design gráfico. Essas áreas são úteis à produção de um currículo efetivo, razão pela qual geralmente indivíduos com habilidades de desenvolvimento de software ou de design gráfico são incluídos como membros das equipes. Cabe ao designer instrucional gerenciar os diferentes atores, com seus respectivos papéis (responsabilidades e atribuições), harmonizando os discursos e guiando a produção da proposta didática [Filatro 2004].

O conteúdo instrucional é parte integrante do DInst, mas não é limitado somente a ele. Sob a ótica da lógica de design, o conteúdo instrucional é um dos elementos compositores do projeto instrucional/educacional. Pode-se compreender como importante elo de comunicação entre o conteudista-educador (professor, tutor, especialista) e o estudante durante a interação no curso, em uma relação dialógica [Garrido, Do Rêgo e Matos 2018].

A concepção de cursos, ocasiões didáticas e artefatos de/para aprendizagem, elaborados sob o suporte do DInst podem ser desenvolvidos por diferentes modelos [ibidem]. Fredetter (2013) e McAndrew \& Scanion (2013) argumentam que no caso de MOOC deve-se considerar os diversos níveis de conhecimento e habilidade dos participantes, bem como a variedade de pontos de vista de um público tão vasto. Para isso, o conteúdo pode ser expandido e modificado ao longo dos momentos de interação para atender às necessidades dos estudantes. Nesse sentido, o design instrucional contextualizado favorece a pluralidade de sujeitos em MOOC [Filatro 2004].

Dentre os recursos de um sistema de MOOC, o fórum de discussão é um elemento essencial para compor o ambiente [Garrido, Do Rêgo e Matos 2018]. O fórum possibilita agregar características de todos os princípios indicados por Scagnoli (2012) para o design instrucional de MOOC. Especialmente nos aspectos de compreensão, engajamento e legado, relacionados com a motivação e interação do estudante. Os princípios de design instrucional para MOOC indicados por Scagnoli (2012) assessoram o design de MOOC. Neste sentido, a saber: experiência prévia (dos estudantes); input (diversidade de perfil dos estudantes); compreensão (acerca das atividades instrucionais realizadas ao longo do curso); engajamento (motivar a interação entre os estudantes); e legado (o conhecimento gerado no ambiente deve ser "herdado" pela próxima geração 
de estudantes).

Ressaltamos que a heterogeneidade dos estudantes em MOOC é um grande desafio para o design instrucional e de interação desses ambientes/cursos. Scagnoli (2012) aponta que a escolha de elementos de design deve considerar a diversidade, propiciando um espaço que inspire e desafie intelectualmente a participação.

\section{Metodologia}

Este estudo ocorreu em quatro etapas: (i) planejamento; (ii) design instrucional do conteúdo do curso; (iii) modelagem do curso/plataforma MOOC utilizando a MoLIC; e por fim, (iv) correção dos diagramas de interação sob a avaliação da modelagem em tempo de design ${ }^{1}$ por meio da MoLVERIC Check (MCheck) [Damian 2016].

A etapa de planejamento consistiu na definição das tecnologias envolvidas na criação da plataforma de MOOC e seus cursos, os colaboradores internos e externos do projeto, na divisão de tarefas da equipe envolvida e no processo de desenvolvimento do conteúdo a ser disponibilizado nos cursos.

Para abrigar os cursos, escolheu-se o ambiente Moodle ${ }^{2}$. Isso ocorreu por ser um ambiente open source e já ser usado em outras iniciativas exitosas de MOOC, como na Fundação Demócrito Rocha ${ }^{3}$ e na Universidade Federal do Rio Grande do Sul (UFRGS), com o Lúmina ${ }^{4}$.

As funcionalidades da plataforma de MOOC e os objetivos educacionais (metas alcançáveis pelos usuários) nortearam a etapa inicial do projeto de design, enfatizando a importância dos objetivos de interação, em consonância com os objetivos de aprendizagem do estudante/usuário. Com isso, foram gerados insights para apoiar a criação dos cenários de uso e roteirizar os diálogos entre os interlocutores da interação, designer e usuário.

$\mathrm{Na}$ etapa de modelagem da interação da plataforma de MOOC, realizada por meio da MoLIC, ocorreu também a construção do curso concomitantemente, para que elementos do design instrucional fossem compreendidos e acrescidos, se necessário, na modelagem da interação do sistema; em atenção aos aspectos pedagógicos que devem estar contidos no design de interação, como avaliações de aprendizagem e percurso pedagógico pretendido, condição sine qua non para integrar o design instrucional ao design de interação.

No caso dos cursos, a modelagem foi genérica sem estar diretamente relacionada ao conteúdo educacional desenvolvido/disponibilizado aos estudantes. Ou seja, a modelagem de interação não está associada a expertise ou conteúdo educacional que será abordado no curso. Desse modo, os possíveis diálogos instrucionais que serão representados ao longo do percurso dos estudantes, bem como a interligação dos fóruns e demais ambientes que fazem parte do sistema (plataforma) são instrumentos dos cursos. Entretanto, apresentamos somente o diagrama de interação que corresponde a

\footnotetext{
${ }^{1}$ Fase de design que antecede a interação do usuário com o sistema. Ou seja, normalmente compreende o período de ideação, planejamento, desenvolvimento e implementação do sistema.

${ }^{2}$ https://moodle.org/

${ }^{3}$ http://ava.fdr.org.br/

${ }^{4}$ https://lumina.ufrgs.br/
} 
modelagem do ambiente MOOC por considerarmos suficiente para explanar acerca da importância da adição dos novos elementos do domínio educacional à MoLIC.

Os pesquisadores/designers envolvidos no desenvolvimento da modelagem foram três estudantes de mestrado em Ciência da Computação, uma estudante de graduação (bolsista de iniciação científica) e um professor-orientador da pós-graduação em Ciência da Computação. Todos participando do mesmo grupo de pesquisa e com experiência em práticas de IHC, como avaliação de comunicabilidade, usabilidade, acessibilidade e design de sistemas (interação, desenvolvimento e implementação) sob a perspectiva do design participativo.

Ressaltamos que após a conclusão dos diagramas MoLIC, ocorreu a avaliação desses diagramas por meio da MCheck [Damian 2016], técnica desenvolvida com o propósito específico de avaliar os diagramas concebidos por meio da MoLIC. Desse modo, foi possível avaliar a qualidade da metacomunicação em tempo de design e revisar os diagramas com base nestas inspeções com a MCheck.

\section{Resultados}

A modelagem da interação de um ambiente educacional interativo sob a ótica da MoLIC permitiu incluir elementos fundamentais focalizados no processo de aprendizagem, como a avaliação do estudante e feedback [Filatro 2008]. Para tanto, alguns princípios de design instrucional foram adotados no desenvolvimento do curso e do sistema de MOOC para alcançar as metas do estudante em sua interação com o ambiente.

O diagrama Avaliação apresentado na Figura 3 é uma versão corrigida, modificada após inspeção para verificação de falhas e erros por intermédio da MCheck [Damian 2016]. Durante a modelagem, percebeu-se a necessidade de adicionar novos elementos, como um diagramático (gráfico) para representar a ação de finalizar a avaliação, simbolizado por um triângulo duplo, denominado de ponto de finalização de avaliação. A finalização de uma avaliação distingue-se do ponto de saída do diálogo, pois o resultado alcançado com a avaliação será armazenado e reverbera nas demais interações do estudante com o curso, em um processo contínuo de diálogo.

$\mathrm{O}$ outro elemento adicionado também tem relação com a avaliação do percurso instrucional do estudante, atuando em correlação com o índice alcançado pelo estudante em suas avaliações. Essa prevenção ativa é representada por [SC] (score capture) e ocorre de modo análogo ao percurso de aprendizagem de um estudante em outros cursos, presenciais ou não. Ou seja, o índice (score) obtido na avaliação é armazenado e influenciará em seu processo de aprendizagem.

Outra característica materializada no diagrama é a possibilidade de repetir a avaliação (cena Repetir avaliação - Figura 3) quantas vezes o estudante achar necessário ou a critério do designer instrucional, a depender da estratégia didática adotada. Essa repetição é indicada pela fala do designer "d: Tentativa X da avaliação", onde $X$ representa a quantidade de repetições indicadas pelo designer instrucional para cada avaliação.

No diagrama Avaliação (Figura 3) há somente uma prevenção de breakdown. O diálogo "Informes sobre a avaliação", presente na cena Descrever Avaliação, é predominantemente composto por signos metalinguísticos, ou seja, apresenta falas 
diretas do designer (normalmente em formato de texto) que contém apontamentos diretos ao estudante (usuário) com intuito de orientação, tanto da avaliação quanto da interação com o sistema. A pretensão é que este diálogo seja concebido pelo designer instrucional (professor, tutor, conteudista) para compor uma prevenção passiva de breakdown ao apresentar a intenção da avaliação e como deverá ser realizada. Além disso, infere-se que situações de ensino-aprendizagem comumente envolvem tentativa e erro.

A cena Avaliação (centro da Figura 3) destaca ainda o efeito perlocutório da repetição da avaliação, indicado diretamente para o estudante ao solicitar a repetição. Descrever Avaliação é a cena que antecipa a descrição da atividade avaliativa. Existe a intenção de que o designer instrucional insira o diálogo adequado na interação para repetir a avaliação, comunicando-se por intermédio de signos metalinguísticos com o estudante.

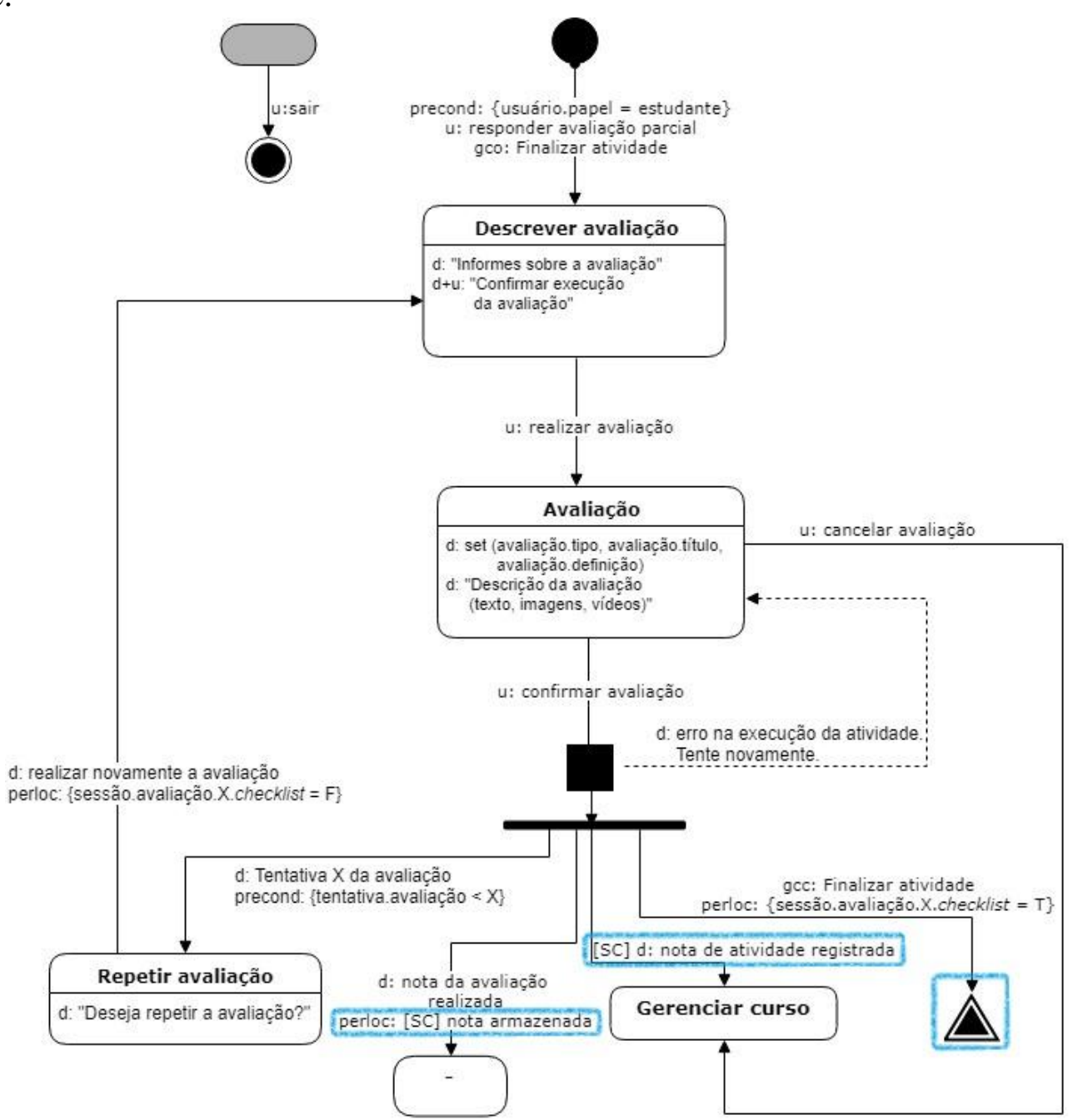

Figura 3: Diagrama MoLIC - Avaliação com os elementos adicionados à MoLIC: triângulo duplo (simbolizando as avaliações) e [SC] (score capture) - prevenção ativa de breakdowns

Outros diagramas foram criados para compor o ambiente MOOC e possuem os novos elementos apresentados neste estudo, como o Fórum de Avaliação e o Fórum Geral. O primeiro é parte integrante da avaliação instrucional do estudante e existirá em cada módulo do curso; representa uma unidade instrucional do conteúdo e os ambientes 
relacionados a ela, como fórum específico e atividades. No diagrama do Fórum Geral é representada a visão geral do momento instrucional e todos os ambientes (demais diagramas) representados em forma mínima, ou seja, as cenas são resumidas para representar melhor a interação entre elas.

\section{Discussão}

O ambiente MOOC concebido no âmbito desta pesquisa focalizou a disponibilização de recursos para fomentar o diálogo entre os pares, como as avaliações por meio de fóruns de discussão e a compreensão do design de ambientes educacionais centrados na troca interpessoal.

Durante todo o processo de design, o foco foi a concepção de um ambiente de MOOC centrado na qualidade da comunicação entre as pessoas durante a interação com o intento de incentivar o diálogo e colaboração durante o percurso instrucional e de interação designer-usuário-sistema. Prezando por inserir nos diagramas as interações, professor-sistema-estudante, sob a perspectiva das boas práticas indicadas no design instrucional.

Articular elementos de áreas distintas, como o design de interação e o design instrucional, foi possível devido aos potenciais elos entre essas duas áreas. Como a compreensão de conversa instrucional (interação estudante-conteúdo) e diálogo didático (interação estudante-educador) pelo design instrucional [Freire 1983; Filatro 2008]. Nesse sentido, é possível alargar essa compreensão ao adotar o conceito de metacomunicação da EngSem e compreender esse diálogo como uma conversa entre designer (educador) e usuário (educando) mediado pelo sistema (MOOC), ou seja, um diálogo educando-sistema-educador (Figura 4).

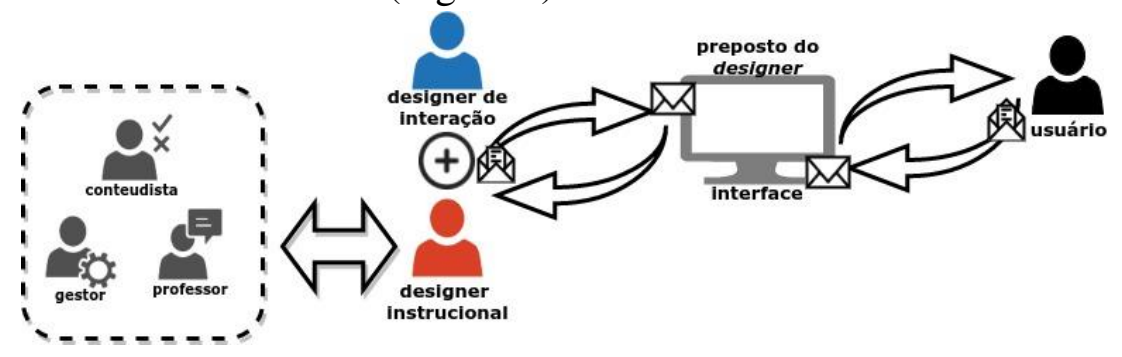

Figura 4 - Metacomunicação designers-sistema-usuário.

A contribuição da MCheck na avaliação dos diagramas de interação foi importante para prevenir possíveis breakdowns durante a fase de desenho, assim não serão apresentados em tempo de interação, ou seja, na interface do sistema em contato com o usuário [Damian 2016].

Um aspecto essencial da adição de novos elementos específicos ao domínio educacional foi a percepção que o ponto de finalização de avaliação (triângulo duplo no diagrama - ver Figura 3) e score captured [SC] tornaram o diálogo mais fluido entre os designers durante a concepção do sistema, possivelmente por refletir no diálogo com o usuário (estudante). Ademais, a concepção dos diagramas é otimizada por meio de elementos que expressam claramente sua intenção e relação com os demais elementos do quadro que é o diagrama como um todo.

Por exemplo, no diagrama de Avaliação ( $c f$. Figura 3) o uso do elemento diagramático ponto de saída ( $c f$. Figura 1) do diálogo não é adequado para lidar com 
uma avaliação instrucional, pois o diálogo não se encerra com a avaliação e sofre influência do resultado da mesma. Nesse caso, foi necessário incluir o elemento ponto de finalização de avaliação para evitar alto número de falas (cenas) resultantes dessa avaliação, permitindo que medidas, especialmente preventivas e geradas pelo sistema, sejam conduzidas pelo professor por indicação do resultado do estudante na avaliação.

O mesmo ocorre com o índice (nota) resultante desta avaliação, denominado de score captured [SC]. Em consonância com o design instrucional, a avaliação é um elemento basilar para o ensino-aprendizagem [Filatro 2004; Filatro 2008]; e tratar o índice alcançado e armazenado como uma prevenção ativa para futuros breakdowns é uma boa prática para sistemas educacionais, seja pela percepção da dificuldade dos estudantes com determinados aspectos do conteúdo ou para indicar um percurso instrucional mais adequado ao indivíduo.

\section{Considerações Finais}

Neste artigo foram apresentados os resultados de uma investigação sobre a adequação da MoLIC à modelagem de uma plataforma de MOOC. Como resultado, identificaram-se limitações dessa ferramenta epistêmica que resultaram na proposição de novos elementos para complementá-la quando aplicada na modelagem da interação de MOOC, em razão do domínio educacional.

Os elementos diagramáticos, score capture [SC] e ponto de finalização de avaliação (representada por um triângulo duplo) ( $c f$. Figura 3), adicionados durante a modelagem com a MoLIC podem ser incorporados em outros projetos similares ou de mesmo domínio. Ressalta-se que esses elementos carecem de uma análise individualizada para atestar sua validade e contribuição para a modelagem de sistemas interativos educacionais, como MOOC.

A principal contribuição com a adição desses elementos, além da adequação da MoLIC para a modelagem de sistemas de domínio educacional, é o favorecimento do diálogo entre designers de formações/atuações diferentes que podem expressar falas específicas com suas intenções de interação para o estudante nesses elementos. Por exemplo, conceber uma avaliação para o estudante em que o resultado desejado no feedback seja a autoria em si e não uma nota alcançada por pontos obtidos por meio de respostas corretas. As falas do professor (designer instrucional) estão presentes diretamente na interface, favorecendo a interpretação do estudante e mitigando falhas na comunicação entre professor-sistema-estudante.

A compreensão sobre a importância da integração de elementos instrucionais na modelagem de sistemas educacionais por meio da MoLIC é apresentada na Figura 4. O estudante (usuário) interage com o ambiente/curso concebido sob orientação da modelagem apresentada neste estudo, sendo que as falas do preposto do designer, a interface, são concebidas como metamensagens conjuntas do designer de interação em articulação com o designer instrucional.

Em trabalhos futuros, pretendemos avaliar a contribuição e impacto desses elementos indicados para modelagem de interação de sistemas específicos do domínio da educação por meio da MoLIC. Além disso, desejamos conduzir a modelagem de interação de forma participativa com profissionais de educação para validar, e 
possivelmente ampliar, os elementos adicionados à MoLIC. Ademais, verificar a adequação da MoLIC na modelagem de outros sistemas de domínio específico, como saúde.

\section{Agradecimentos}

Agradecemos aos membros do SPIDeLab (Semio-Participatory Interaction Design Lab), ao Grupo de Pesquisa e Extensão em Informática, Educação e Sociedade - Onda Digital, à Coordenação de Aperfeiçoamento de Pessoal de Nível Superior (CAPES) pelo financiamento desta pesquisa, sob os códigos \#88887.464930/2019-00 DS e \#88882.453912/2019-01 DS e ao Conselho Nacional de Desenvolvimento Científico e Tecnológico $(\mathrm{CNPq})$ pela Bolsa de Produtividade em Pesquisa.

\section{Referências}

Aguaded, I. (2013). The MOOC Revolution: A new form of education from the technological paradigm?. Comunicar: Revista Científica de Comunicación y Educación. v. 21, n. 41, p. 07-08.

Araujo, A. C. I. C. (2008). Apoio ao design e à interpretação de modelos de interação humano-computador representados em MoLIC. Departamento de Informática, Pontifícia Universidade Católica do Rio de Janeiro, PUC-RIO, Rio de Janeiro.

Barbosa, S.; Silva, B. (2010). Interação humano-computador. Elsevier Brasil.

Carey, K. (2012). Into the future with MOOCs. The Chronicle of Higher Education. v. 59, n. 2, p. 29.

Carvalho, A. P. de; Pereira, F. H. S; Assunção, O. B; Pereira, A. F; Prates, R. O. (2019). An analysis of MoLIC's consolidation. In Proceedings of the 18th Brazilian Symposium on Human Factors in Computing Systems (IHC '19). Association for Computing Machinery, New York, NY, USA, Article 11,1-15.

Damian, A. L. (2016). Técnicas para inspeção de diagramas MOLIC. Master's thesis. Universidade Federal do Amazonas, Amazonas, Brasil. Dissertação de mestrado.

De Souza, C. S. (2005). The semiotic engineering of human-computer interaction. MIT press.

De Souza, C. S. e Leitão, C. F. (2009). Semiotic engineering methods for scientific research in hci. Synthesis Lectures on Human-Centered Informatics, v. 2, n. 1, p. $1-122$.

Do Rêgo, B. B; Garrido, F. A; Matos, E. (2017). Identifying influences of the quality of interaction on dropout rates of mooc: preliminary results. In Simpósio Brasileiro de Informática na Educação - SBIE. p. 1766-1768.

Do Rêgo, B. B; Garrido, F. G; Rosa, J; Matos, E. (2019). Communicability problems on MOOCs: a study around metacommunication. In Simpósio Brasileiro de Informática na Educação - SBIE. p. 1261-1270.

Freire, P. (1983). Extensão ou Comunicação? 8ª Ed., Rio de Janeiro: Paz e Terra.

Filatro, A. C. (2004). Design instrucional contextualizado-educação et. Senac.

Filatro, A. (2008). Design instrucional na prática. São Paulo: Pearson Education do 
Brasil.

Fredette, M. (2013). How to convert a classroom course into a MOOC. Campus Technology. v. 8, n. 28, p. 13.

Garrido, F. A; Do Rêgo, B; Matos, E. (2018). Modelando a Interação (Humano-Computador) de um Fórum de Discussão para MOOC: MoLIC em uso. RENOTE - Revista Novas Tecnologias na Educação. v. 16, n. 2, p. 321-330.

Martin, F. G. (2012). Will massive open online courses change how we teach?. Communications of the ACM. v. 55, n. 8, p. 26-28.

McAndrew, P; Scanlon, E. (2013). Open learning at a distance: lessons for struggling MOOCs. Science. v. 342, n. 6165, p. 1450-1451.

Melo, A. M; Saldanha, J. de Freitas; e Wernz, M. C. G. (2012). Desafios à pesquisa em Computação em contexto educacional-qualidade no uso de objetos de aprendizagem em perspectiva. In Anais do Workshop de Desafios da Computação Aplicada à Educação. p. 60-69.

Paula, M. G. (2003). Projeto da interação humano-computador baseado em modelos fundamentados na engenharia semiótica: construção de um modelo de interação. Programa de Pós-graduação em Informática do Departamento de Informática da PUC-Rio, PUC-Rio, Rio de Janeiro.

Prates, R. O; De Souza, C. S; Barbosa, S. D. J. (2000). Methods and tools: a method for evaluating the communicability of user interfaces. Interactions. v. 7, n. 1, p. 31-38.

Scagnoli, N. I. (2012). Instructional Design of a MOOC. Proceedings of the 2016 Conference Academy of Human Resource Development Jacksonvillle. Disponivel em: $<$ https://www.ideals.illinois.edu/bitstream/handle/2142/44835/Instructional\%20Desig n\%20of\%20a\%20MOOC.pdf>

Silva, Bruno S. (2005). MoLIC Segunda Edição: revisão de uma linguagem para modelagem da interação humano-computador. Master's thesis. Pontifícia Universidade Católica do Rio de Janeiro.

Silva, B. S. da; Barbosa, S. D. J. (2007). Designing human-computer interaction with MoLIC diagrams-a practical guide. Monografia em Ciência da Computação, PUC-Rio.

Zhao, Y; Wang, A; Sun, Y. (2020). Technological environment, virtual experience, and MOOC continuance: A stimulus-organism-response perspective. Computers \& Education. v. 144, n. 103721, p. 1-14. 